tetracyclines; and were identified as serotype 5 by specific rabbit antiserum. The pathogenicity of human ureaplasmas is still debated although some can cause non-specific urethritis." This case shows that they can at least produce disease in patients with depressed immunity.

The arthritis in this case should not be confused with more chronic types of monoarthritis and polyarthritis that occur in patients with hypogammaglobulinaemia. ${ }^{4}$ Fluid aspirated from an acutely inflamed joint in such patients should be cultured for bacteria and mycoplasmas, including ureaplasmas. The results of culture and antibiotic sensitivity testing may not be available for several days so intravenous tetracycline treatment should start immediately since only about $10 \%$ of human ureaplasmas are insensitive to tetracyclines. Prolonged tetracycline treatment over some months is probably necessary.

We thank Mr J B King, Mr L Lowe, and Mr P D Sutarir for their help with the surgical management of this patient.

${ }^{1}$ Foy, H M, et al, Journal of Infectious Diseases, 1973, 127, 388.

${ }^{2}$ MacLeod, A D, Furr, P M, and Taylor-Robinson, D, British fournal of Venereal Diseases, 1976, 52, 337.

3 Taylor-Robinson, D, Csonka, G W, and Prentice, M J, Quarterly fournal of Medicine, 1977, 46, 309.

${ }^{4}$ Webster, A D B, et al, British Medical fournal, 1976, 1, 1314.

${ }_{5}$ Taylor-Robinson, D, et al, fournal of General Microbiology, 1971, 68, 97.

(Accepted 25 October 1977)

Divisions of Immunological Medicine and Communicable Diseases,

Clinical Research Centre, Northwick Park Hospital, Harrow, Middlesex HA1 3UJ

A D B WEBSTER, MRCP, consultant physician

D TAYLOR-ROBINSON, MD, FRCPATH, consultant microbiologist

P M FURR, FIMLS, research assistant

G L ASHERSON, DM, FRCP, consultant physician

\section{Rheumatoid stricture of oesophagus}

Lesions of the upper alimentary tract and pathological changes in oesophageal vessels in rheumatoid arthritis have been described, ${ }^{1}$ as have rheumatoid nodules of the pharynx..$^{2}$ Rheumatoid stricture of the oesophagus, however, has apparently not been observed. We describe such a case.

\section{Case report}

A 46-year-old housewife had suffered from rheumatoid arthritis from the age of 19. Two pregnancies had produced some remission, but seven years before the present admission she had had a fracture of the right femoral neck, which had been treated by internal fixation. Since that time she had been chairbound. She had suffered from dysphagia for two years. On admission she had tight atrophic skin, and advanced arthritic changes of the hands, knees, and ankles. There were no visible signs of rheumatoid vascular changes or neuropathy. Her haemoglobin was $9.6 \mathrm{~g} / \mathrm{dl}$. This had been fluctuating between 6-9 $\mathrm{g} / \mathrm{dl}$ according to her records. The sheep cell agglutination test was positive at $1 / 32$, and the latex slide test was positive. Tests for antinuclear factor were not done. A barium swallow had shown a stricture in the mid-oesophagus and this had been treated by periodic dilatations at another hospital. Latterly, these had become difficult and she could swallow only liquids. Repeat barium studies showed a tight stricture $3 \mathrm{~cm}$ long at the level of the aortic arch; the rest of the oesophagus was normal and no hiatal hernia was seen. Oesophagoscopy confirmed a severe stricture at $20 \mathrm{~cm}$ with mucosal ulceration and slough formation. Overnight $\mathrm{pH}$ recordings with an electrode in the oesophagus $5 \mathrm{~cm}$ above the diaphragm showed no acid reflux. In view of the increasing difficulty in dilating this stricture, the age of the patient, and the progressive malnutrition, we decided that resection should be attempted. Total oesophagectomy with cervical oesophagogastric anastomosis was therefore performed and she made an uneventful recovery, except for a persistently raised blood urea concentration, possibly due to renal lesions from her rheumatoid disease.

The resected oesophagus showed mucosal ulceration over an area of $4 \mathrm{~cm}$ in the middle third, and the mucosa on either side of this was normal. The mucosa below the stricture was definitely squamous and not columnar. Histological examination (figure) showed an extensive area of shallow ulceration extending into the submucosa lined by necrotic tissue and inflamed granulation tissue with focal lymphoid aggregates. The adjacent

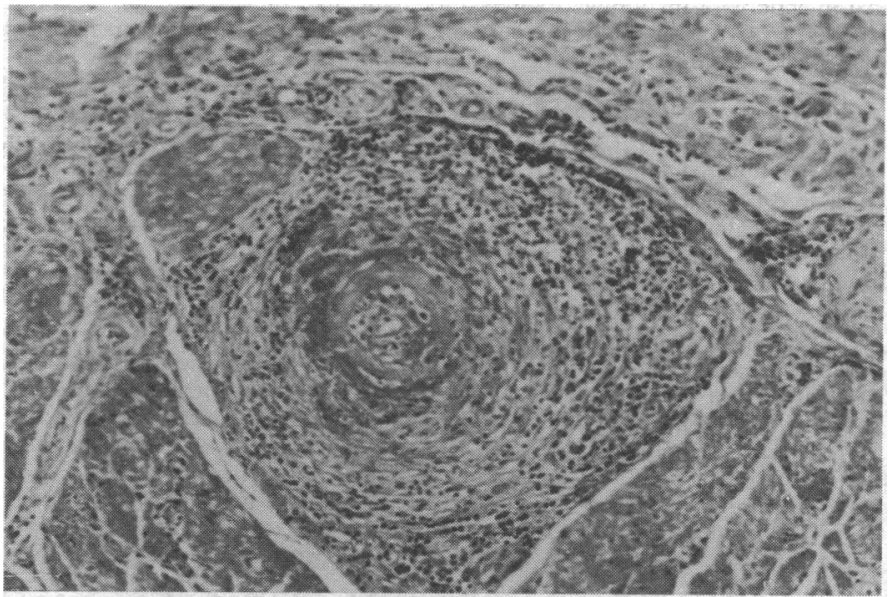

Rheumatoid nodule in excised oesophagus

squamous epithelium was hyperplastic. Within the submucosa and muscularis propria were two arteries showing a severe arteritis with complete destruction of the walls with fibrinoid necrosis and lymphoid infiltration. Other smaller arteries showed endothelial proliferation with calcification and obliteration of the lumen. The muscularis propria was hypertrophic and there was submucosal fibrosis. The ulceration and fibrosis appeared to be secondary to the arteritic process and, in view of the long history of rheumatoid arthritis, it was considered to be rheumatoid.

\section{Comment}

Benign stricture of the middle third of the oesophagus has a variety of causes including columnar-epithelium-lined oesophagus, gastro-oesophageal reflux, corrosive injury, and involvement of the oesophagus in mediastinal disease such as tuberculosis. From the information obtained at operation and pathological examination of the resected oesophagus, clearly none of these causes could have been present in our patient. In addition, the histological evidence of unequivocal arterial occlusion due to arteritis suggested that this stricture had developed on the basis of local ischaemia from a rheumatoid arteritis. We could not find any record of this development in rheumatoid arthritis.

\section{Requests for reprints to HRM.}

1 Cruickshank, B, Annals of the Rheumatic Diseases, 1954, 13, 136.

${ }^{2}$ Raven, R W, et al, Annals of the Rheumatic Diseases, 1948, 7, 63.

${ }^{3}$ McInnes, G T, and Littman, C D, British Medical fournal, 1977, 1, 685.

(Accepted 26 October 1977)

Queen Elizabeth Hospital, Birmingham B15 2TH

V JOHN, FRCSED, thoracic surgical registrar

A J STIRLING, $\mathrm{MB}$, CHB, house physician

H R MATTHEWS, FRCS, consultant thoracic surgeon

\section{Exercise and insulin absorption from subcutaneous tissue}

Exercise-induced hypoglycaemia in insulin-dependent diabetics has been attributed primarily to an increase in the peripheral tissue uptake of glucose. ${ }^{12}$ We postulate instead that exercise enhances the absorption of insulin from the subcutaneous site of its injection.

\section{Materials, methods, and results}

Serial glucose and immunoreactive insulin (IRI) concentrations were measured in five healthy volunteers after $(a)$ exercise alone; $(b)$ injection of $10 \mathrm{IU}$ of mono-component Actrapid insulin followed by complete rest; 
and $(c)$ injection of $10 \mathrm{IU}$ of mono-component insulin followed by exercise. All injections of insulin were given subcutaneously on the anterior aspect of the right thigh. The subjects were exercised intermittently for 60 minutes on a bicycle ergometer. During the last 30 minutes of the "exercise" experiments the subjects were rested. On the "rest" days the subjects sat in deep armchairs for the entire 90 minutes after the injection of insulin. Insulin concentrations in serum were measured by radioimmunoassay (using a kit from the Radiochemical Centre, Amersham). Glucose concentrations were measured by an autoanalyser.

Exercise alone induced an initial fall in blood glucose concentration by 15 to $20 \%$; this fall was not associated with any significant alteration in serum IRI concentration. Exercise alone did not induce any consistent change in IRI concentrations at any stage (figure). In the resting subject insulin concentration became maximal 60 minutes after the injection. The blood glucose concentration did not alter significantly. When the injection of insulin was followed by exercise, serum IRI concentration increased sharply at 10 minutes and achieved a maximum at 20 minutes; the peak IRI concentration was twice as high as that achieved when the subjects were rested after the insulin injection. After exercise, the IRI concentration was significantly higher throughout the experimental period of 90 minutes.

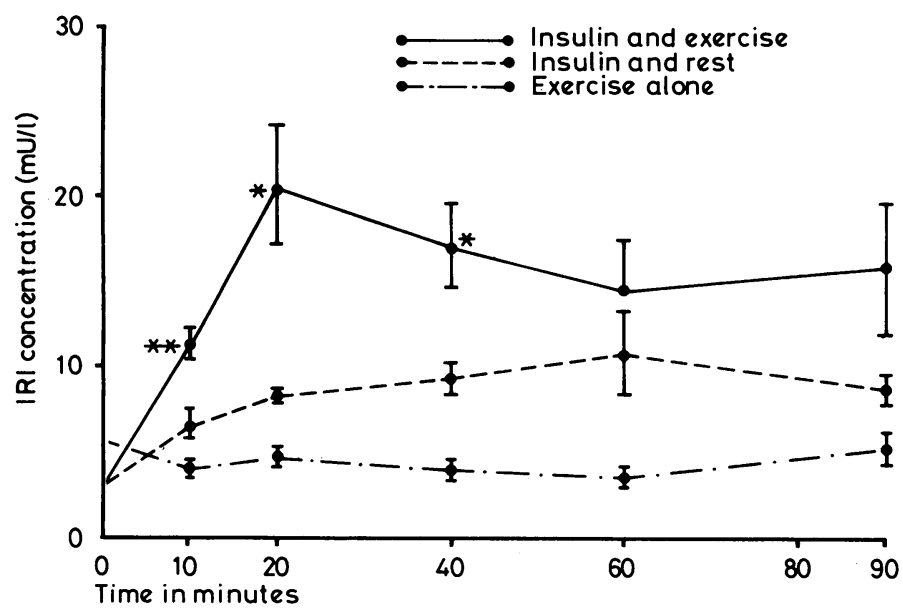

Effect of exercise on immunoreactive insulin (IRI) concentration in four normal subjects (mean $\pm \mathrm{SE}$ ). The fifth patient was overweight and hyperinsulinaemic but the effect of exercise in her was similar.

${ }^{*} \mathrm{P}<0.05{ }^{* *} \mathrm{P}<0.01$.

\section{Discussion}

The fact that serial IRI concentrations were much higher after exercise than those associated with rest must mean that exercise increases the rate and the magnitude of insulin absorption from subcutaneous tissue. Since exercise alone did not alter serial IRI concentration significantly, increased endogenous secretion cannot account for our observations. This difference was maintained at 90 minutes; this would imply that the effect of exercise may outlast the duration of exercise. Such an increase in absorption of insulin from subcutaneous tissue after exercise provides an obvious and a more satisfactory explanation for exercise-induced hypoglycaemia in insulin-dependent diabetics than some other circulating hypoglycaemic factor. ${ }^{3}$

No significant hypoglycaemia was observed in any of the subjects; this is probably the result of a combination of a small insulin dose; switching off of endogenous insulin secretion; and the release of glucagon, catecholamines, and cortisol. In diabetic patients who lack an adequate B-cell reserve much larger doses of insulin are used and when after exercise a large bolus of insulin is absorbed into the circulation little is achieved by switching off the limited endogenous secretion of insulin. These patients thus have only their glucagon and catecholamine secretion to protect them from hypoglycaemia.

The mechanism underlying the enhanced absorption of insulin from subcutaneous tissue is probably through an increase in blood flow in the injected limb. Binder ${ }^{4}$ measured the absorption of insulin after an intramuscular injection and concomitantly measured the local blood flow; in a group of 12 patients he studied no correlation between insulin absorption and local blood flow was found. Binder did not, however, measure the effect of an increase in local blood flow on insulin absorption in the same person. On the other hand, Binder ${ }^{4}$ showed quite convincingly that the rate of insulin absorption is not altered by increasing the skin temperature.
Exercise thus augments the rate and magnitude of insulin absorption from subcutaneous tissue and this effect may play an important part in the pathogenesis of exercise-induced hypoglycaemia in the insulin-dependent diabetic patient. Exercise does not probably alter the requirement of insulin to the same extent as it enhances the bioavailability of subcutaneously injected insulin. It would be of interest to investigate the possibility that patients may be protected from exercise-induced hypoglycaemia by injecting insulin into those parts of the body which are not likely to be exercised or to study the effect of such drugs as would prevent exercise-induced enhancement of insulin absorption.

We thank Mrs A Winsbury for secretarial help.

${ }^{1}$ Marble, A, in Joslin's Diabetes Mellitus, p 287. Philadelphia, Lea and Febiger, 1971 .

${ }^{2}$ Nikkila, E, et al, Diabetes, 1968, 17, 209.

${ }^{3}$ Goldstein, M S, On the Nature and Treatment of Diabetes, ed B S Leibel and Wrenshall p 308. Excerpta Medica Foundation, Amsterdam, 1965. ${ }^{4}$ Binder, C, Absorption of Injected Insulin. Copenhagen, Munksgaard, 1969.

(Accepted 31 October 1977)

Royal Free Hospital, London NW3 2QG

P DANDONA, MRCP, DPHIL, senior medical registrar

D HOOKE, MB, senior house officer

$\mathrm{J}$ BELL, top grade biochemist

\section{Remission of psoriasis during haemodialysis}

Increased epidermal cell proliferation is a characteristic feature of psoriasis. The factors responsible may derive from the epidermis, dermis, or blood. We present here evidence that the factors involved are blood-borne. Two patients with chronic renal failure and psoriasis noticed complete clearing of their skin lesions during treatment with haemodialysis.

\section{Case reports}

Case 1-A 57-year-old man developed psoriasis at the age of 25 . Six years later he presented with back pain and was shown to have polycystic disease of the kidneys. During the years leading up to end-stage renal failure he had noticed some seasonal fluctuation in the extent of his psoriasis; otherwise it had been chronic and stable. In November 1975, just before starting haemodialysis, his psoriasis was extensive on the trunk, elbows, knees, and scalp. Within eight weeks of treatment with dialysis his psoriasis cleared. He continued on dialysis and in October 1977 his psoriasis was confined to two tiny papules in the scalp.

Case 2-A 50-year-old man developed psoriasis on the elbows and knees in 1949 and progressively lesions of the scalp in 1960 and on the legs and trunk in early 1971 . In November 1971 he presented with ankle oedema and the diagnosis of chronic proliferative glomerulonephritis was established; his renal function rapidly deteriorated and haemodialysis was instituted. Within three weeks of starting dialysis his widespread and extensive psoriasis vanished. In April 1974 he had a cadaver renal transplant and was started on immunosuppressive treatment with prednisolone $40 \mathrm{mg}$ and azathioprine $150 \mathrm{mg}$ daily. Three weeks later he was taken off dialysis. The first sign of recurrence of his psoriasis, mild disease of the scalp and trunk, occurred three weeks after dialysis was stopped. One year later, after gradual reduction of immunosuppressive treatment to prednisolone $15 \mathrm{mg}$ and azathioprine $150 \mathrm{mg}$ daily, his psoriasis relapsed further with moderately severe disease of the scalp, discoid lesions on the trunk, and guttate papules on the limbs.

\section{Comment}

Remission of psoriasis during haemodialysis was first reported in 1976. ${ }^{1}$ The patient described had chronic renal failure and psoriasis; the psoriasis was cleared for the first time within two weeks of starting haemodialysis; remission was maintained throughout 12 months' dialysis, and for a further 11 months after successful renal transplantation and the introduction of immunosuppressive treatment; a trivial relapse then finally occurred when the patient fractured a tibia. ${ }^{2}$ 